\title{
Smoothed Particle Hydrodynamics for Electrophysiological Modeling: an Alternative to Finite Element Methods
}

\author{
Èric Lluch ${ }^{1,2(\bowtie)}$, Rubén Doste ${ }^{1}$, Sophie Giffard-Roisin ${ }^{3}$, Alexandre This $^{2,4}$, \\ Maxime Sermesant ${ }^{3}$, Oscar Camara ${ }^{1}$, Mathieu De Craene ${ }^{2}$, and \\ Hernán G. Morales ${ }^{2}$ \\ 1 PhySense, ETIC, Universitat Pompeu Fabra, Barcelona, Catalonia \\ ${ }^{2}$ Medisys, Philips Research, France \\ eric.lluch@philips.com \\ 3 Université Côte d'Azur, Inria, France \\ ${ }^{4}$ Inria Paris, France
}

\begin{abstract}
Finite element methods (FEM) are generally used in cardiac 3D-electromechanical modeling. For FEM modeling, a step of a suitable mesh construction is required, which is non-trivial and time-consuming for complex geometries. A meshless method is proposed to avoid meshing. The smoothed particle hydrodynamics (SPH) method was used to solve an electrophysiological model on a left ventricle extracted from medical imaging straightforwardly, without any need of a complex mesh. The proposed method was compared against FEM in the same left-ventricular model. Both FEM and SPH methods provide similar solutions of the models in terms of depolarization times. Main differences were up to $10.9 \%$ at the apex. Finally, a pathological application of SPH is shown on the same ventricular geometry with an added scar on the heart wall.
\end{abstract}

Keywords: SPH, Meshless, FEM, Cardiac electrophysiology

\section{Introduction}

Patient-specific modeling has become an interesting research topic in the cardiac electrophysiology community because it can help to understand the electrical propagation and its pathologies [2]. FEM is a well-established numerical approach often used to investigate the electro-mechanics of the human heart $[3,13]$. The generation of complex meshes is necessary. Meshing is one of the main bottlenecks for the clinical translation of cardiac modeling tools since it is difficult to have a streamlined and automated pipeline to generate accurate FE simulations from imaging data [10]. Another non-trivial step of FEM in electro-mechanics is the coupling between electrophysiology and mechanics when meshes with different resolution for both problems are used. It is expected that a way to overcome these difficulties could be through a meshless approach.

Various meshless methods have demonstrated the ability to provide a computational feasible model for cardiac electrophysiology simulations, without burden 
mesh generation $[2,5,15]$. In this paper, $\mathrm{SPH}[8]$ is proposed to numerically solve the Mitchell-Schaeffer (MS) electrophysiological model [7] on the electrical depolarization of the left ventricle. To evaluate the accuracy of this approach, a comparison with a FEM implementation [6] was conducted. Finally, a scar was added to the ventricular myocardium to show the potential use of the proposed meshless approach in a pathological case. The goal is to explore the accuracy, speed and limitations of SPH with respect to FEM, as a first step towards a potential full electro-mechanical heart model using a meshless approach.

\section{Method}

In this section, the electrophysiological model and the SPH discretization scheme are explained. For further details of the FEM approach, refer to [6].

\subsection{Electrophysiological Model}

In this paper, the macroscopic biophysical mono-domain model Mitchel-Schaeffer together with a diffusion term [13] was used to model the cardiac electrophysiology. This model was chosen because it captures the action potential duration (APD) (Fig. 1), considers fiber orientation in the diffusion term and is only governed by 6 parameters, which might facilitate a more precise model personalization since less parameters need to be fitted to given data.

$$
\left\{\begin{array}{l}
\partial_{t} v=\frac{w v^{2}(1-v)}{\tau_{\text {in }}}-\frac{v}{\tau_{\text {out }}}+I_{\text {app }} \\
\partial_{t} w=2 \begin{cases}\frac{1-w}{\tau_{\text {open }}} & \text { if } v<v_{\text {gate }} \\
\frac{-w}{\tau_{\text {close }}} & \text { if } v>v_{\text {gate }}\end{cases}
\end{array}\right.
$$

When considering the geometry, a diffusion term $\operatorname{div}(C \nabla v)$ is required to the first Equation (1) [13]. $C \in \mathbb{R}^{3,3}$ is the connectivity tensor defined as

$$
C=(\boldsymbol{\tau} \otimes \boldsymbol{\tau}(1-a r)+I d \cdot a r) \cdot c,
$$

with $\tau \in \mathbb{R}^{3}$ being the vector corresponding to the fiber orientation, $\otimes$ the tensor product, $I d \in \mathbb{R}^{3,3}$ the identity matrix, ar $\in \mathbb{R}$ the anisotropic ratio and $c \in \mathbb{R}$ the conductivity coefficient that controls the propagation velocity. ar controls the conduction velocity in the fiber orientation with respect to the transverse plane, e.g. in the case $a r=1$, the fiber orientation is not anymore taken into account, hence reducing the model to the isotropic case.

The parameters $\tau_{\text {in }}, \tau_{\text {out }}, \tau_{\text {open }}, \tau_{\text {close }} \in \mathbb{R}$ control the duration of the four stages of the APD. The depolarization phase is controlled by $w \in \mathbb{R}$ and $v_{\text {gate }} \in \mathbb{R}$ defines at which point the APD starts. $I_{\text {app }} \in \mathbb{R}$ corresponds to the first stimulus of the transmembrane potential $v \in \mathbb{R}$. 

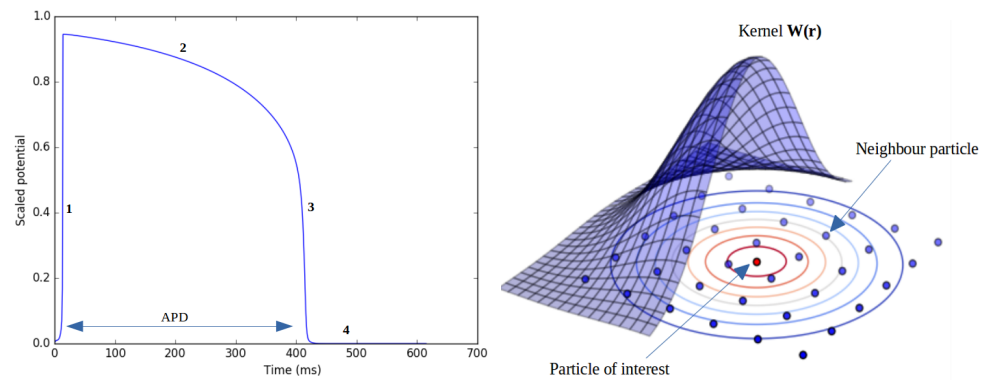

Fig. 1: Left: Example of the 4 stages of the cardiac action potential: initiation (1), plateau (2), decay (3), and recovery (4). Right: Example of an SPH Kernel for $2 \mathrm{D}$.

\subsection{SPH Discretization}

$\mathrm{SPH}$ is a meshless Lagrangian method, where each solid particle carries its own properties such as density, conductivity, etc. Given a continuous function $f$ : $\mathbb{R}^{3} \rightarrow \mathbb{R}$ representing a particle property at the spatial position $\boldsymbol{r}$, it can be approximated with a delta Dirac function (3):

$$
f(\boldsymbol{r})=(f * \delta)(\boldsymbol{r})=\int_{\mathbb{R}^{3}} f\left(\boldsymbol{r}^{\prime}\right) \delta\left(\boldsymbol{r}-\boldsymbol{r}^{\prime}\right) \mathrm{d} \boldsymbol{r}^{\prime} \approx \int_{\mathbb{R}^{3}} f\left(\boldsymbol{r}^{\prime}\right) W\left(\boldsymbol{r}-\boldsymbol{r}^{\prime}, h\right) \mathrm{d} \boldsymbol{r}^{\prime} .
$$

Notice that the delta Dirac function was approximated with a kernel function $W\left(\boldsymbol{r}-\boldsymbol{r}^{\prime}, h\right)$, where $h \in \mathbb{R}$ is the so-called smoothing length (Fig. 1). For Equation (3) to hold, $W$ must fulfill the following

$$
\int_{\mathbb{R}^{3}} W\left(\boldsymbol{r}-\boldsymbol{r}^{\prime}, h\right) \mathrm{d} \boldsymbol{r}^{\prime}=1 \text { and } \lim _{h \rightarrow 0} W\left(\boldsymbol{r}-\boldsymbol{r}^{\prime}, h\right)=\delta\left(\boldsymbol{r}-\boldsymbol{r}^{\prime}\right) .
$$

The integral in (4) is approximated as a finite sum, where the density $\rho_{j}$ and the mass $m_{j}$ are obtained by replacing the infinitesimal volume $\mathrm{d} \boldsymbol{r}^{\prime}$ by the finite volume (5).

$$
\begin{aligned}
f(\boldsymbol{r}) & =\lim _{h \rightarrow 0} \int_{\mathbb{R}^{3}} \frac{f\left(\boldsymbol{r}^{\prime}\right)}{\rho\left(\boldsymbol{r}^{\prime}\right)} W\left(\boldsymbol{r}-\boldsymbol{r}^{\prime}, h\right) \rho\left(\boldsymbol{r}^{\prime}\right) \mathrm{d} \boldsymbol{r}^{\prime} \\
& \propto \sum_{j=1}^{N} m_{j} \frac{f_{j}}{\rho_{j}} W\left(\boldsymbol{r}_{i}-\boldsymbol{r}_{j}, h\right)=f_{i},
\end{aligned}
$$

where $f_{i}$ is the approximated value of the function $f$ at the position $\boldsymbol{r}$, i.e. at the particle of interest $i$. Due to the previous formulation (5) the derivative of the function $f$ in the same position $\boldsymbol{r}$ can be approximated as a derivative of the kernel function (6) 


$$
\nabla f_{i}=\sum_{j} m_{j} \frac{f_{j}}{\rho_{j}} \nabla W\left(\boldsymbol{r}_{i}-\boldsymbol{r}_{j}, h\right)
$$

The electrophysiological model (1) in the SPH formulation reads:

$$
\begin{aligned}
\partial_{t} v_{i} & =C_{i} \circ \sum_{j=1}^{n_{i}} m_{j} \frac{v_{j}-v_{i}}{\rho_{j}} \nabla^{2} W\left(\boldsymbol{r}_{i}-\boldsymbol{r}_{j}, h\right) \\
& +\nabla v_{i} \operatorname{div}\left(C_{i}\right)+\frac{w_{i} v_{i}^{2}\left(1-v_{i}\right)}{\tau_{\text {in }}}-\frac{v_{i}}{\tau_{\text {out }}}+I_{\text {app }, i} \\
\partial_{t} w_{i} & =2 \begin{cases}\frac{1-w_{i}}{\tau_{\text {open }}} & \text { if } v_{i}<v_{\text {gate }} \\
\frac{-w_{i}}{\tau_{\text {close }}} & \text { if } v_{i}>v_{\text {gate }},\end{cases}
\end{aligned}
$$

where $\circ$ is an element wise multiplication (Hadamard product), $\nabla^{2} W \in \mathbb{R}^{3,3}$ is the Hessian matrix of the kernel including all the Hessian derivatives and

$$
\nabla v_{i} \operatorname{div}\left(C_{i}\right)=\sum_{j=1}^{n_{i}} m_{j} \nabla W\left(\boldsymbol{r}_{\boldsymbol{i}}-\boldsymbol{r}_{\boldsymbol{j}}, h\right) \frac{C_{j}-C_{i}}{\rho_{j}} \cdot \sum_{j=1}^{n_{i}} m_{j} \frac{v_{j}-v_{i}}{\rho_{j}} \nabla W\left(\boldsymbol{r}_{\boldsymbol{i}}-\boldsymbol{r}_{\boldsymbol{j}}, h\right),
$$

where $n_{i}$ is the number of neighbors of the particle $p_{i}$.

Boundary conditions are difficult to handle in SPH, even when simple boundary conditions such as symmetric surface boundary are required. This is due to the truncation of the particle neighborhood near a boundary, which results in a truncation of the integral of equation (5) [4]. Moreover, when it is assumed that the system connectivity only changes because the particles lose or gain connectivity through a boundary (no-flux), there is no need to place special conditions on the gradient of the potential function $v$ near the boundary $[4,8]$. In other words, if all the boundaries fulfill the no-flux condition, then the symmetry of the SPH ensures that the system conserves its flux because the particles interact amongst themselves. On top of this, a corrective smoothed particles method (CSPM) was implemented to overcome the lack of particles in the boundaries while enhancing the solution accuracy inside the domain [4]. After applying CSPM, the discretization scheme has an accuracy of $O\left(h^{2}\right)$ for interior points and $O(h)$ for points near or on the boundary, where $h$ is the distance between particles. The distance depends on the choice of the spatial resolution.

The cubic B-spline kernel (9) was used here since its first derivatives are positive for neighbor particles close to the particle of interest [4]

$$
W\left(\boldsymbol{x}-\boldsymbol{x}^{\prime}, h\right)=\frac{\alpha_{d}}{h^{3}} \begin{cases}1-\frac{3}{2} q^{2}+\frac{3}{4} q^{3} & \text { for } q<1 \\ \frac{1}{4}(2-q)^{3} & \text { for } 1 \leq q<2 \\ 0 & \text { elsewhere }\end{cases}
$$

where in order to fulfill (4), the coefficient $\alpha_{d}=\frac{1}{\pi}$ and $q$ is defined as 


$$
q=\frac{\left|\boldsymbol{x}-\boldsymbol{x}^{\prime}\right|}{h}=\frac{\boldsymbol{r}}{h} .
$$

Regarding the time integration scheme, a forward explicit Euler method was used for SPH whose accuracy is $O(h), h$ being the time step. For FEM, the modified Crank-Nicholson / Adams-Bashforth (MCNAB) was used [13].

\section{$3 \quad$ Experiments}

To evaluate the proposed SPH-based electrophysiological model, the same model was solved with a FEM scheme and results were compared for the electrical depolarization. An image-based left-ventricular geometry was evaluated in this work to have a preliminary comparison between the methods. The two methods are labeled as:

- FEM $_{\text {MS }}$ : Mitchell-Schaeffer model discretized with FEM [7].

- SPH MS: Mitchell-Schaeffer model discretized with SPH.

For FEM and SPH approaches, two different resolutions were considered. The low resolution had 18667 nodes and the high resolution had 51037 nodes for both approaches. For $\mathbf{F E M}_{\mathbf{M S}}$, a tetrahedral mesh was computed from the segmented left ventricle. For the proposed approach $\mathbf{S P H} \mathbf{H}_{\mathbf{M S}}$, a set of equidistributed points from the same anatomy was used. Each SPH particle has a density of 1053 $\mathrm{kg} / \mathrm{m}^{3}$, corresponding to reported myocardium density [14]. The mass of each particle was computed as the product of the density times the volume of the cubic cell defined between the particle of interest and the neighboring particles. To understand the impact of key intrinsic parameters of the $\mathbf{S P H}_{\mathbf{M S}}$, additional experiments were conducted for several kernel sizes in these two resolutions. In order to evaluate the accuracy of these experiments, an analysis of the $L^{2}$ differences between FEM and SPH activation times, as well as the computational time, were investigated (Table 1).

For all simulations, myocardial fiber orientation was included in each of the nodes to achieve a physiological behavior. Fibers were assigned following the rule-based model angles described by D. Streeter et al. [12]. Regarding the parameters, an initial electrical impulse $I_{\text {app }}=-580000 \frac{\mathrm{mV}}{\mathrm{s}}$ was imposed in a set of points on the apex surface corresponding to $80 \mathrm{~mm}^{2}$ during $4 \mathrm{~ms}$ so that in the first time step with an integration time of $d t=10^{-4} \mathrm{~s}$ an initial potential of $v=-58 \mathrm{mV}$ was obtained. Time variables were $\tau_{\text {open }}=120 \mathrm{~ms}$, $\tau_{\text {close }}=150 \mathrm{~ms}, \tau_{\text {in }}=0.3 \mathrm{~ms}, \tau_{\text {out }}=6 \mathrm{~ms}$, following [7]. An anisotropic ratio $a r=0.16$ was used.

Moreover, a scar was added in the myocardium of the same left ventricle to show how SPH handles a pathological example. In particular, the electrical activation was simulated during one second for both healthy and pathological scenarios. It was assumed that the heart rate was $75 \mathrm{bpm}$, i.e. the heart period was $0.8 \mathrm{~s}$. Under this assumption, three activation phases were observed within one simulated second: the depolarization phase, where particles get activated; the 
repolarization phase, where particles get deactivated; the second depolarization phase, where particles get activated again. The scar was placed in the septalanterior region close to the base. Shape and location of the induced scar are shown in Fig. 2. The high resolution (51037 particles) model with a kernel size of $3 \mathrm{~mm}$ was used for both healthy and pathological simulations. The scar tissue was applied to 1621 particles while 5891 particles were treated as gray tissue (tissue near the scar). The rest of the particles were considered as healthy tissue. Two different pathological experiments with different model parameters were simulated. In the first pathological simulation, denoted as 'pathological with low conductivity', particles in the gray zone and in the scar were modified according to [1], in such a way that their conductivity coefficient is reduced but not null. In the second pathological case, denoted as 'pathological with zero conductivity', particles in the scar are assumed to have zero conductivity.

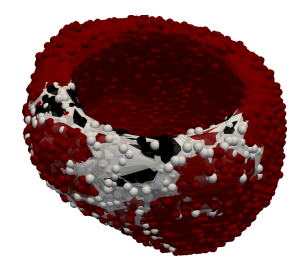

Fig. 2: Scar regions in black, Grey zones in grey and healthy zones in red.

To visualize the discretized domains using both discretization schemes, the structure of the meshes is shown in Fig. 3. In the case of SPH, a 3D Delaunay filter from Paraview (Kitware Inc., Clifton Park, USA) was used to enhance the volume visualization, since unconnected points in $3 \mathrm{D}$ do not provide a good visual 3D representation.

\section{Results and Discussion}

Results section is structured as follows: first, a sensitivity analysis of the impact of key intrinsic SPH parameters is presented. Then, a qualitative comparison against the FEM solution for depolarization is presented. Finally, experiments to show the $\mathbf{S P H}_{\mathrm{MS}}$ applicability in a pathological case with a scar are shown. Depolarization and repolarization phases were compared between healthy and pathological cases using SPH.

\subsection{Sensitivity Analysis}

A sensitivity analysis of particle resolution, as well as the kernel size was conducted for SPH and compared against FEM results. Five kernel sizes ranging from 0.003 to $0.007 \mathrm{~m}$ were evaluated. A kernel size $<0.0025 \mathrm{~m}$ fails due to insufficient number of neighbors, while a kernel size $>0.007 \mathrm{~m}$ has so many 
neighbors that the computational time was excessive for the potential gain in accuracy.

Table 1: $L^{2}(\mathbb{R})$ norm of the difference of depolarization times between SPH and FEM simulations in the endocardium and computational time (in brackets) of 150 ms with a 4 processor Intel computer for both SPH and FEM.

\begin{tabular}{|c|c|c|c|c|c|c|}
\hline Number of & \multicolumn{5}{|c|}{ SPH kernel size } & \multirow{2}{*}{ FEM } \\
\cline { 2 - 7 } particles & 0.003 & 0.004 & 0.005 & 0.006 & 0.007 & \\
\hline \multirow{2}{*}{18667} & 10.294 & 9.792 & 9.767 & 9.500 & 10.911 & - \\
& $(37 s)$ & $(1 m 13 s)$ & $(3 m 13 s)$ & $(6 m 43 s)$ & $(10 m 43 s)$ & $(9.92 s)$ \\
\hline \multirow{2}{*}{51037} & 4.258 & 4.209 & 4.723 & 5.103 & 6.082 & - \\
& $(4 m 52 s)$ & $(11 m 03 s)$ & $(21 m 02 s)$ & $38 m 41 s)$ & $(57 m 26 s)$ & $(27.41 s)$ \\
\hline
\end{tabular}

Table 1 shows that the $L^{2}$ error is not linear neither over the kernel size nor over the resolution. A reduction of the difference between SPH and FEM was observed when the number of degrees of freedom was incremented for the evaluated kernel sizes. In terms of computational time, it increases linearly over the kernel size and faster over the degrees of freedom. The FEM implementation was faster than the SPH one. A GPU implementation (relatively easy with SPH formulation) could overcome this disadvantage of the SPH approach [9]. The choice of a kernel size of $3 \mathrm{~mm}$ and a resolution of 51037 particles is a good balance between kernel size and number of degrees of freedom since the $L^{2}$ error with respect to FEM is very small and it was relatively fast to compute. For the rest of the results, this choice of kernel size and resolution was used.

\subsection{Qualitative Comparison}

Depolarization times were first qualitatively compared using a discrete colormap divided by ten isochronous on the ventricle. In all simulations the electrical activation started from the apex until the septal base. $\mathbf{S P H} \mathbf{M S}_{\mathbf{S}}$ and $\mathbf{F E M} \mathbf{M S}_{\mathbf{M S h}}$ sw the same range of depolarization times and a similar activation pattern (Fig. 3). Moreover, it is observed that all particles in the endocardium get activated after $123 \mathrm{~ms}$. To evaluate the propagation with both approaches, a picture of the activation times for the same cross section in both approaches is shown in Fig. 3 (middle column). In general, a visual comparison of this figure shows that the behavior over the whole ventricular volume is similar for both FEM and SPH methods. The left ventricle endocardium was then mapped into a disk by the use of Quasi-conformal mapping (QCM) [11] to better visualize differences in all regions. The mapped results are shown in the bullseye plots of Fig. 3.

To insight into the differences between $\mathbf{S P H}_{\mathbf{M S}}$ and $\mathbf{F} \mathbf{E} \mathbf{M}_{\mathbf{M S}}$, the absolute differences between the depolarization times of these two numerical approaches were computed on the endocardium. These differences were projected on a homeomorphic disk as shown in Fig. 4. In this figure, it is observed that the highest 

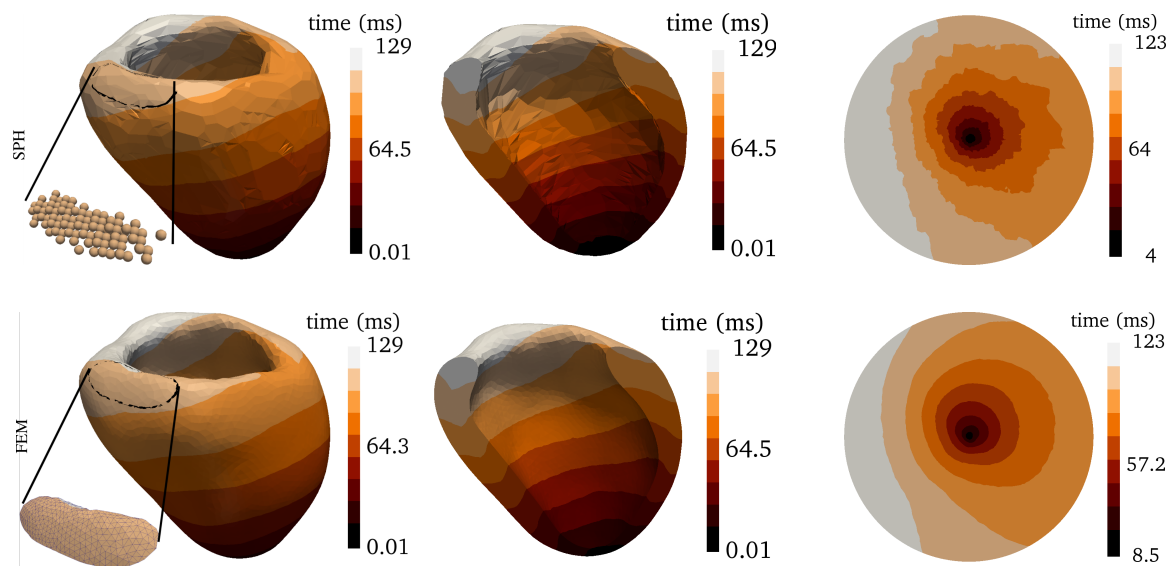

Fig. 3: Left: Contour color map of depolarization times for the left ventricle model. Middle: a longitudinal cross section of the ventricle. Right: projected endocardium onto a disk for both SPH and FEM simulations.

differences of depolarization times in the endocardium occur near the apex with a peak difference of $13.5 \mathrm{~ms}$. For most of the domain, the differences are less than $4 \mathrm{~ms}$ as can be seen in the histogram of Fig. 4.
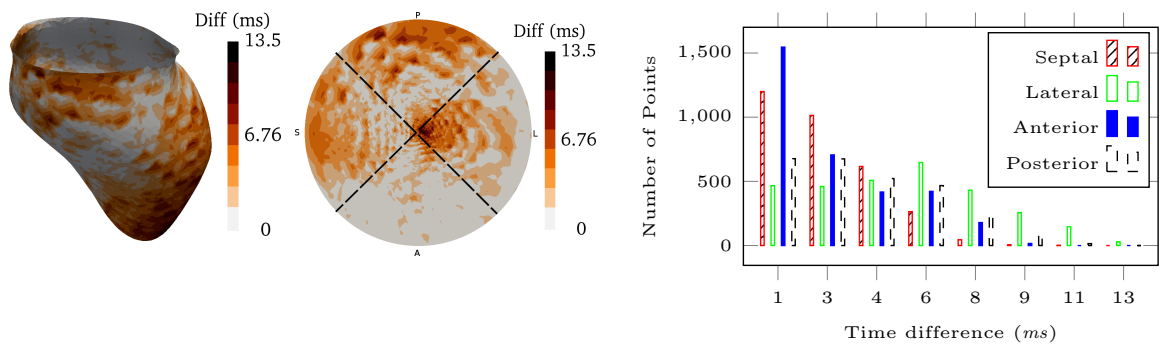

Fig. 4: Left: Difference map of depolarization times between $\mathbf{S P H}_{\mathbf{M S}}$ and $\mathbf{F E M}_{\mathbf{M S}}$. Middle: projection of the differences onto a disk divided into septal $(\mathbf{S})$, lateral $(\mathbf{L})$, anterior $(\mathbf{A})$ and posterior $(\mathbf{P})$ regions. Right: histogram of number of points per region with respect to the time difference.

\subsection{Pathological case}

Results of the experiments described in Section 3 for both healthy and pathological cases are shown in this subsection. The pathological simulations took $22 \mathrm{~m} 45 \mathrm{~s}$ for $99 \mathrm{~ms}$. Depolarization times, repolarization times and second depolarization 
times are presented using a discrete colormap divided by ten isochronous on the ventricle (Fig. 5). Results for the pathological case with zero conductivity were thresholded at $0.66 \mathrm{~s}$ to avoid having particles at two repolarization phases simultaneously, which facilitates the comparison among the first phases for the three experiments (healthy, pathological with low conductivity and pathological with zero conductivity).
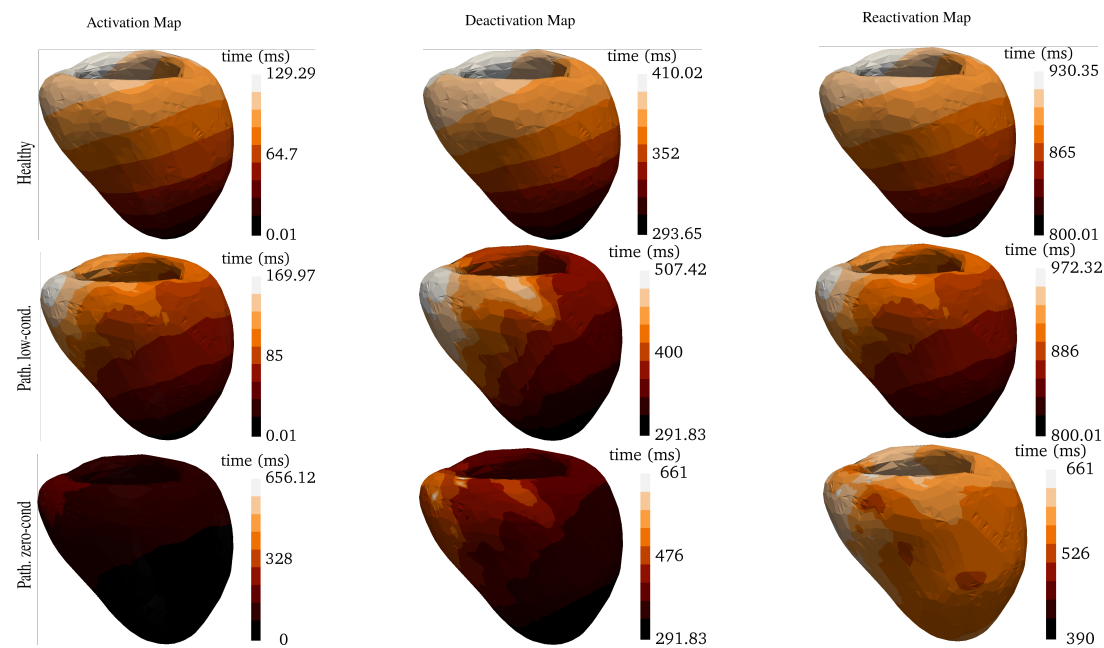

Fig. 5: Different phases for each of the three simulations (from above to below): healthy, pathological with low conductivity and pathological with zero conductivity on the scar).

In pathological cases, some regions of the ventricle take a longer time to get activated due to the low conductivity around the scar. In fact, the latest particle gets activated at $169.97 \mathrm{~ms}$ (low conductivity) and $656.12 \mathrm{~ms}$ (zero conductivity) for the two pathological scenarios, whereas in the healthy case, the latest particle does it after $129 \mathrm{~ms}$. However it was observed for all three cases, that the ventricle gets repolarized from the apex to the base. In the two pathological cases, the activation pattern goes around the geometry of the scar. In the middle column of Fig. 5, the depolarization phase is depicted. For all cases, the heart starts its depolarization from the apex as it should be. Nevertheless, the depolarization times for the pathological cases are higher due to the lower conductivity in the gray and scar zones. Similarly to the repolarization time, the pattern for the deactivation in the pathological cases takes the shape of the scar into account.

Finally, the second repolarization is shown on the right of Fig. 5. In a heart without arrhythmia, the second repolarization phase should have the same pattern as the first repolarization phase when the electrical impulse is given in the apex again after $0.8 s$. Nevertheless, for the pathological case with zero conductivity, particles in the gray zone get reactivated before $0.8 \mathrm{~s}$ due to their low 
(but not zero) conductivity. In particular, the first particle in the gray zone to be activated (at $390 \mathrm{~ms}$ ) forces the apex to reactivate much earlier than $0.8 \mathrm{~s}$. This implies that the heart gets reactivated before it should, which is known as 'reentry arrhythmia' and has been observed in patients with scars.

Finally, as part of the limitation of this study, full heart geometries will be considered in the future to evaluate the robustness of the proposed $\mathbf{S P H}$ Ms Moreover, the validation of SPH for electrophysiology should be performed by comparing it with patient data or with a higher number of validated synthetic geometries. The impact of the particle distribution on the results needs to be revised as well, especially when particle motion is taken into account.

\section{Conclusions}

In this paper, it was shown that $\mathrm{SPH}$ is an alternative method to model cardiac electrophysiology. This work has not only demonstrated that the presented meshless method can provide a physiological meaningful model, but that the results are similar to existing mesh-based methods in terms of activation patterns and depolarization times. The comparison shows promising results towards a proper validation of the method and accuracy assessment against real data. Moreover, a pathological case was also investigated to show the potential use of $\mathrm{SPH}$ in the present of a scar. SPH methods are a promising alternative to produce patient-specific simulations. Their ability to import an unstructured set of points without any mesh makes the integration of sparse imaging data (including anatomy and velocities) straightforward.

\section{Acknowledgements}

The work is supported by the European Union Horizon 2020 research and innovation programme under grant agreement No 642676 (CardioFunXion). The authors would like to thank the organizers of this project: Bart Bijnens and Mathieu De Craene. Finally, the authors would also like to thank David-Soto Iglesias for all the help provided with the conformal mapping of the endocardium.

\section{References}

1. R. Cabrera Lozoya. Radiofrequency ablation planning for cardiac arrhythmia treatment using modeling and machine learning approaches. Theses, Université Nice Sophia Antipolis, September 2015.

2. J.O. Campos, R.S. Oliveira, R.W. dos Santos, and B.M. Rocha. Lattice boltzmann method for parallel simulations of cardiac electrophysiology using \{GPUs\}. Journal of Computational and Applied Mathematics, 295:70 - 82, 2016. \{VIII\} Pan-American Workshop in Applied and Computational Mathematics.

3. R. Chabiniok, V. Y. Wang, et al. Multiphysics and multiscale modelling, datamodel fusion and integration of organ physiology in the clinic: ventricular cardiac mechanics. Interface focus, 6(2):20150083, 2016. 
4. J. K. Chen, J. E. Beraun, and T. C. Carney. A corrective smoothed particle method for boundary value problems in heat conduction. International Journal for Numerical Methods in Engineering, 46(2):231-252, 1999.

5. P. Chinchapatnam, K. Rhode, et al. Voxel based adaptive meshless method for cardiac electrophysiology simulation. In Lecture Notes in Computer Science (including subseries Lecture Notes in Artificial Intelligence and Lecture Notes in Bioinformatics), volume 5528, pages 182-190, 2009 .

6. S. Marchesseau, H. Delingette, et al. Fast parameter calibration of a cardiac electromechanical model from medical images based on the unscented transform. Biomechanics and Modeling in Mechanobiology, 12(4):815-831, 2013.

7. C. C. Mitchell and D. G. Schaeffer. A two-current model for the dynamics of cardiac membrane. Bulletin of Mathematical Biology, 65(5):767-793, 2003.

8. J. J Monaghan. Smoothed particle hydrodynamics. Reports on progress in physics, 68(8):1703, 2005

9. D. Nishiura, M. Furuichi, and H. Sakaguchi. Computational performance of a smoothed particle hydrodynamics simulation for shared-memory parallel computing. Computer Physics Communications, 194:18-32, 2013.

10. N. Smith, A. de Vecchi, et al. euHeart: personalized and integrated cardiac care using patient-specific cardiovascular modelling. Interface Focus, 1(3):349-364, jun 2011.

11. D. Soto-Iglesias, C. Butakoff, et al. Integration of electro-anatomical and imaging data of the left ventricle: An evaluation framework. Medical Image Analysis, 32:131-144, aug 2016

12. D. D. Streeter, H. M. Spotnitz, et al. Fiber orientation in the canine left ventricle during diastole and systole. Circulation Research, 24(3):339-347, 1969.

13. H. Talbot, S. Marchesseau, et al. Towards an interactive electromechanical model of the heart. Interface Focus, 3(2), 2013.

14. T. Yipintsoi, P. D. Scanlon, et al. Density and water content of dog ventricular myocardium. Proceedings of the Society for Experimental Biology and Medicine. Society for Experimental Biology and Medicine (N.Y.), 141(3):1032-5, dec 1972.

15. H. Zhang, L. Wang, P. J. Hunter, and S. Pengcheng. Meshfree framework for imagederived modelling. In 2008 5th IEEE International Symposium on Biomedical Imaging: From Nano to Macro, pages 1449-1452. IEEE, may 2008. 